\title{
Fortified Extract of Red Berry, Ginkgo biloba, and White Willow Bark in Experimental Early Diabetic Retinopathy
}

\author{
Claudio Bucolo, Giuseppina Marrazzo, Chiara Bianca Maria Platania, Filippo Drago, \\ Gian Marco Leggio, and Salvatore Salomone \\ Department of Clinical and Molecular Biomedicine, Section of Pharmacology and Biochemistry, University of Catania, \\ Viale Andrea Doria 6, 95125 Catania, Italy \\ Correspondence should be addressed to Claudio Bucolo; claudio.bucolo@unict.it
}

Received 16 March 2013; Accepted 7 May 2013

Academic Editor: Ghulam Mohammad

Copyright (C) 2013 Claudio Bucolo et al. This is an open access article distributed under the Creative Commons Attribution License, which permits unrestricted use, distribution, and reproduction in any medium, provided the original work is properly cited.

Diabetic retinopathy is a complex condition where inflammation and oxidative stress represent crucial pathways in the pathogenesis of the disease. Aim of the study was to investigate the effects of a fortified extract of red berries, Ginkgo biloba and white willow bark containing carnosine and $\alpha$-lipoic acid in early retinal and plasma changes of streptozotocin-induced diabetic rats. Diabetes was induced by a single streptozotocin injection in Sprague Dawley rats. Diabetics and nondiabetic (control) rats were treated daily with the fortified extract for the ten days. Retina samples were collected and analyzed for their TNF- $\alpha$ and VEGF content. Moreover, plasma oxidative stress was evaluated by thiobarbituric acid reacting substances (TBARS). Increased TNF- $\alpha$ and VEGF levels were observed in the retina of diabetic rats. Treatment with the fortified extract significantly lowered retinal cytokine levels and suppressed diabetes-related lipid peroxidation. These data demonstrate that the fortified extract attenuates the degree of retinal inflammation and plasma lipid peroxidation preserving the retina in early diabetic rats.

\section{Introduction}

Diabetic retinopathy, a diabetes-related complication, is the leading cause of blindness and visual impairment in workingage individuals [1]. Diabetic retinopathy is a chronic disease that develops in stages and is rarely detected in the first few years of diabetes. The incidence of the disease increases to $50 \%$ by 10 years and to $90 \%$ by 25 years of diabetes [1].

Oxidative stress appears to be an important feature of the diabetic complications such as retinopathy. Apart from the well-known increase in lipid peroxide, diabetics have lower concentrations of erythrocyte glutathione and have higher concentrations of dehydroascorbate in their plasma and lower levels of vitamin E in their platelets. Oxidative stress causes a production of chemically reactive molecules, which induce a variety of proinflammatory mediators such as VEGF and TNF- $\alpha[2,3]$. The earliest changes detectable in diabetic retinopathy are loss of pericytes, capillary basement membrane thickening, edema, and formation of microaneurysms. These structural and functional changes are followed by microvascular occlusion, neovascularization, and neurodegeneration [4].

Considering that oxidative stress and inflammation represent the key factors in the onset and progression of diabetic retinopathy, antioxidant and anti-inflammatory products are expected to produce significant therapeutic advantages.

Current treatments associated with antidiabetic drugs are mostly intended to regulate vascular changes, inflammation, and the increased oxidative stress. Dietary supplements have been shown to play an important role in ameliorating clinical signs of diabetes [5]. Many studies have identified flavonoids that are associated with a reduction in the risk of advanced retinal degeneration. Recently [6], it has been demonstrated that eriodictyol, a strong antioxidative flavonoid extracted from Eriodictyon californicum, significantly reduces the retinal levels of VEGF, ICAM-1, TNF- $\alpha$, and eNOS in diabetic rats.

Much evidence supports the roles of berry extracts, $\alpha$-lipoic acid, and L-carnosine as antioxidant and antiinflammatory products $[5,7,8]$. It has been demonstrated 
that supplementation with these compounds can inhibit retinal diabetes-induced abnormalities [5]. In particular, $\alpha$-lipoic acid can scavenge free radicals and can act as an antioxidant [9]. Alpha-lipoic acid acts both directly, by radical quenching and metal chelation, and indirectly through the recycling of other antioxidants such as ascorbic acid, vitamin E, and glutathione [9]. Alpha-lipoic acid has been shown to protect against cataract formation $[10,11]$, a diabetic complication resulting from polyol accumulation, and it is beneficial also in a rat model of diabetic retinopathy [12]. Alpha-lipoic acid attenuates apoptosis in the retinal capillary endothelial cells of rats and decreases the levels of oxidative stress markers such as 8-hydroxydeoxyguanosine (8-OHdG) and nitrotyrosine [13]. Furthermore, Kowluru et al. [14, 15] demonstrated that its supplementation completely prevents the diabetesinduced increase in nitrotyrosine and activation of NF- $\kappa \mathrm{B}$, while decreasing the levels of VEGF in the rat retina $[14,15]$. Multiple biochemical pathways that are known to increase the production of reactive oxygen species (ROS), advanced glycosylation end products (AGEs), and reactive nitrogen species (RNS) have been linked to hyperglycemia/diabetesinduced vascular injury [16].

L-Carnosine, a dipeptide primarily produced in skeletal muscle and the central nervous system, acts both as a scavenger of ROS and as an inhibitor of AGE production [8]. L-carnosine is one of the most abundant antioxidants in the brain and retina [8]. Carnosine is synthesized from $\beta$ alanine and histidine and is specifically degraded by selective enzymes such as carnosinase-1 (CN-1). It has been suggested that the carnosine/carnosinase system plays an important role in the pathogenesis of diabetic complications [8]. Red berries are rich in phenolic compounds as well as many other essential nutritional components, such as flavonoids and phenolic acids, which have a wide range of beneficial properties, including retinal protection [17]. Epidemiologic evidence suggests that a high consumption of flavonoids may be useful against coronary heart disease, stroke, and neurodegenerative disorders $[18,19]$. The high level of scavenging activity of red berry extracts toward chemically generated reactive oxygen species has been described in several studies [18]. Salix alba (white willow bark) extract is used for antiinflammatory medical treatments due to its ability to suppress prostaglandin synthesis. The main component of Salix alba is salicin, an analogue of the widely used acetyl salicylic acid [20]. Two trials investigating the effects of Salix alba found evidence that daily doses standardized to $120 \mathrm{mg}$ or $240 \mathrm{mg}$ of salicin were better than placebo for short-term improvements in pain and rescue medication [20,21].

Ginkgo biloba leaf extract (GBE) contains many different flavone glycosides and terpenoids [22]. It is well known that GBE has an antioxidant action as a free radical scavenger, and an anti-inflammatory effect suppressing the production of active oxygen and nitrogen species [22]. GBE inhibits the increase in the products of the oxidative decomposition of low-density lipoprotein (LDL), reduces the cell death in various types of neuropathy, and prevents the oxidative damage to mitochondria, suggesting that its beneficial effects on neurodegenerative diseases are related to prevention of chronic oxidative damage [23].
In the present study, we investigated the effect of systemic treatment with a fortified extract (FE) on proinflammatory mediators (TNF- $\alpha$ and VEGF), in the diabetic rat retina. Moreover, we evaluated plasma oxidative stress by measuring the thiobarbituric acid reacting substances (TBARS) [24].

\section{Materials and Methods}

2.1. Animals and Reagents. Male Sprague Dawley rats (approximately $200 \mathrm{~g}$ ) were obtained from Charles River (Calco, Italy). All the animals were treated according to the ARVO Statement for the Use of Animals in Ophthalmic and Vision Research and the Directive 2010/63/EU of the European Parliament and of the Council. The animals were fed on standard laboratory food and were allowed free access to water in an air-conditioned room with a $12 \mathrm{~h}$ light/ $12 \mathrm{~h}$ dark cycle. Final group sizes for all measurements were $n=8-$ 10. STZ was purchased from Sigma-Aldrich (St. Louis, MO, USA). All other reagents were purchased from standard commercial suppliers unless otherwise noted.

2.2. Induction of Diabetes and Treatment Schedule. STZ acts by producing concentrations of peroxides greater than can be tolerated by the islets of Langerhans, since these are poor in glutathione peroxidase. The induction of diabetes was performed as previously described [24]. Briefly, the animals received a single injection (iv) of STZ $(60 \mathrm{mg} / \mathrm{kg})$. Control (nondiabetic) animals received the vehicle alone. After $24 \mathrm{~h}$, animals with blood glucose levels greater than $250 \mathrm{mg} / \mathrm{dL}$ were considered diabetic and randomly divided into groups. All experiments were carried out 10 days after induction of diabetes. We confirmed the diabetic state by evaluating glycemia daily using a blood glucose meter (Accu-Check Active; Roche Diagnostic, Milan, Italy). A group of rats were treated with the FE extract intraperitoneally (i.p.) starting from $30 \mathrm{~min}$ after STZ administration. Treatment consisted of daily injections with the blend suspension containing the following: $300 \mathrm{mg} / \mathrm{kg} \alpha$-lipoic acid, $150 \mathrm{mg} / \mathrm{kg}$ Salix alba extract (containing 15\% salicin), $100 \mathrm{mg} / \mathrm{kg}$ berry extract (35\% polyphenols; $6 \%$ anthocyanins), and $65 \mathrm{mg} / \mathrm{kg}$ Ginkgo biloba extract (22.0-27.0\% Ginkgo flavonoids; $5.0-7.0 \%$ terpene lactones; ginkgolic acid content $<5 \mathrm{ppm}$ ), and $50 \mathrm{mg} / \mathrm{kg}$ L-carnosine (Tiomax, Sooft Italia SpA, Montegiorgio, Italy). We chose these concentrations because they represent the dose recommended by an ophthalmologist in clinical practice [25]. After 10 days animals were killed, and retina and blood samples were collected to assess cytokines (TNF- $\alpha$ and VEGF) and TBARS, respectively.

2.3. Measurements of TNF- $\alpha$ and VEGF. After 10 days from the STZ injection, the eyes were enucleated and retinal samples were collected; each retina was handled as previously described [24]. Briefly, the retinal samples were homogenized in $100 \mu \mathrm{L}$ of cocktail solution supplemented with protease inhibitors before use. Samples were centrifuged, and protein levels were measured (Mini BCA Kit; Pierce Scientific, CA, USA). TNF- $\alpha$ and VEGF protein levels were estimated with commercial ELISA kits. The tissue sample concentration was 
calculated from a standard curve and corrected for protein concentration.

2.4. Lipid Peroxidation Assay. Lipid peroxidation is defined as "the oxidative deterioration of polyunsaturated lipids," that is, lipids that contain more than two carbon-carbon double covalent bonds. As previously described [6], plasma lipid peroxidation was assessed by the thiobarbituric acid reacting substances method. The thiobarbituric acid test is one of the most frequently used tests for measuring the peroxidation of fatty acids. Briefly, plasma was mixed with hydrochloric acid and thiobarbituric acid, incubated and heated for $20 \mathrm{~min}$, and then deproteinated with trichloroacetic acid. The absorbance of the malonaldehyde and thiobarbituric acid pink product was detected at $532 \mathrm{~nm}$. The results are expressed in $\mathrm{nmol}$ MDA per $\mathrm{mL}$ of plasma.

\section{Results}

3.1. Glycemia and Body Weight. Ten days after onset of diabetes, blood glucose values in diabetic rats treated with FE were significantly higher than corresponding values in nondiabetic rats (Table 1). FE does not interfere with glycemia values in nondiabetic rats (data not shown). Body weights of diabetic rats treated with FE were significantly less than those of nondiabetic rats but were not different compared with the diabetic group (Table 1).

3.2. TNF- $\alpha$ and VEGF. Figure 1 shows the retinal TNF- $\alpha$ and VEGF levels. Experimental diabetes significantly increases the TNF- $\alpha$ level (from $3.8 \pm 0.5 \mathrm{pg} / \mathrm{mg}$ to $9.7 \pm 1.0 \mathrm{pg} / \mathrm{mg} ; P<$ $0.001)$. FE treatment significantly reduced the retinal levels of TNF- $\alpha$ in the STZ-treated group (from $9.7 \pm 1.0 \mathrm{pg} / \mathrm{mg}$ to $4.5 \pm 0.3 \mathrm{pg} / \mathrm{mg} ; P<0.001$ ). We also assessed the effect of FE treatment on VEGF content. As shown in Figure 1, STZ-treated animals showed a 2-fold increase in VEGF levels (from $7.5 \pm 2.5 \mathrm{pg} / \mathrm{mg}$ to $14.9 \pm 2.0 \mathrm{pg} / \mathrm{mg} ; P<0.001$ ). $\mathrm{FE}$ treatment in diabetic rats significantly lowered VEGF levels compared with control animals (from $14.9 \pm 2.0 \mathrm{pg} / \mathrm{mg}$ to $8.7 \pm 1.5 \mathrm{pg} / \mathrm{mg} ; P<0.001)$. The FE did not significantly affect cytokine levels in normal nondiabetic rats (data not shown).

3.3. Lipid Peroxidation. A significant increase in plasma lipid peroxidation was observed after 10 days of diabetes (Figure 2$)$. FE treatment significantly $(P<0.001)$ suppressed diabetes-related lipid peroxidation $(1.9 \pm 0.2 \mathrm{MDA} \mathrm{nmol} / \mathrm{mL}$ and $3.8 \pm 0.1 \mathrm{MDA} \mathrm{nmol} / \mathrm{mL}$, resp.). The FE did not significantly affect lipid peroxidation in normal nondiabetic rats (data not shown).

\section{Discussion}

The present results show that FE of red berry, Ginkgo biloba and white willow bark, containing $\alpha$-lipoic acid and Lcarnosine, may blunt some of the negative effects due to hyperglycemia, such as oxidation, inflammation and VEGF expression, which are the main causes of diabetic retinopathy.
TABLE 1: Effects of STZ-induced diabetes on body weight and blood glucose levels after 10 days. Control (nondiabetic) group is rats injected with only the vehicle used to dissolve STZ. FE was given intraperitoneally for 10 days. Diabetes was induced by $60 \mathrm{mg} / \mathrm{kg}$ (i.v.) injection of STZ.

\begin{tabular}{lcc}
\hline Groups & Body weight $(\mathrm{g})$ & Blood glucose $(\mathrm{mg} / \mathrm{dL})$ \\
\hline Control & $215 \pm 15$ & $98 \pm 10$ \\
Diabetic & $170 \pm 12^{*}$ & $360 \pm 30^{* *}$ \\
Diabetic + FE & $180 \pm 20^{*}$ & $380 \pm 15^{* *}$ \\
\hline
\end{tabular}

Data are expressed as mean \pm SD.

${ }^{*} P<0.01,{ }^{* *} P<0.0001$ versus control; $(n=8-10)$.

Diabetes is characterized by a progressive vascular impairment mediated by pericyte loss that leads to an increase of retinal leakage and macular edema and the formation of new vessels [26]. Many studies demonstrated that VEGF and TNF- $\alpha$ contribute to the progression of diabetic retinopathy and that their expression is increased in the diabetic retina. The role of VEGF in the development of diabetic complications in the eye is well established, whereas the role of TNF- $\alpha$ is still under investigation. TNF- $\alpha$ has been implicated in the pathogenesis of diabetic retinopathy, this proinflammatory cytokine induces expression of endothelial adhesion molecules via activation of NF- $\kappa$ B. This latter is able to increase the expression of other inflammatory mediators such as cyclooxygenase enzyme-2 (COX-2). This enzyme may also be activated by glycosylation products [27]. In the present study, we observed that TNF- $\alpha$ levels in the diabetic rat retina were significantly higher in comparison with retina obtained from the control group (Figure 1(a)). According to our data, other groups demonstrated that retinal TNF- $\alpha$ is significantly elevated in diabetic rats $[28,29]$. TNF- $\alpha$ is a proinflammatory cytokine mainly generated by inflammatory cells and activated endothelial cells. This cytokine is a potent inducer of the leukostasis elicited by other actors such as VEGF, IL- $1 \beta$, and PAF in the retinal vasculature [30]. As reported by Joussen et al. [31] TNF- $\alpha$ is one of the most important cytokines in diabetic retinopathy both involved in leukocyte activation and in endothelial cell apoptosis. Further, as showed by the same authors $[29,31]$, TNF- $\alpha$ receptor inhibitor and nonsteroidal anti-inflammatory drugs attenuated leukocyte adhesion in diabetic retinal vessels as well as the retinal leakage, suggesting a key role of TNF- $\alpha$.

Our results, beside confirming that TNF- $\alpha$ levels are increased in the retina of STZ-induced diabetic rats, show that FE treatment prevents the increase in TNF- $\alpha$ levels (Figure 1(a)), suggesting that the antioxidant treatment improved the inflammatory milieu of the diabetic retina.

To further investigate whether this FE had any effect on VEGF levels in our model of early diabetic retinopathy, we also measured VEGF levels in retina.

VEGF is a growth factor, which stimulates angiogenesis, promotes vascular permeability, and induces dissociation of tight junctions. Production of VEGF is elicited by high glucose levels, AGEs, IGF-I, angiotensin II, and hypoxia; all these factors increase in the retinal diabetic microvascular bed [32]. Moreover, VEGF levels have been found to be 


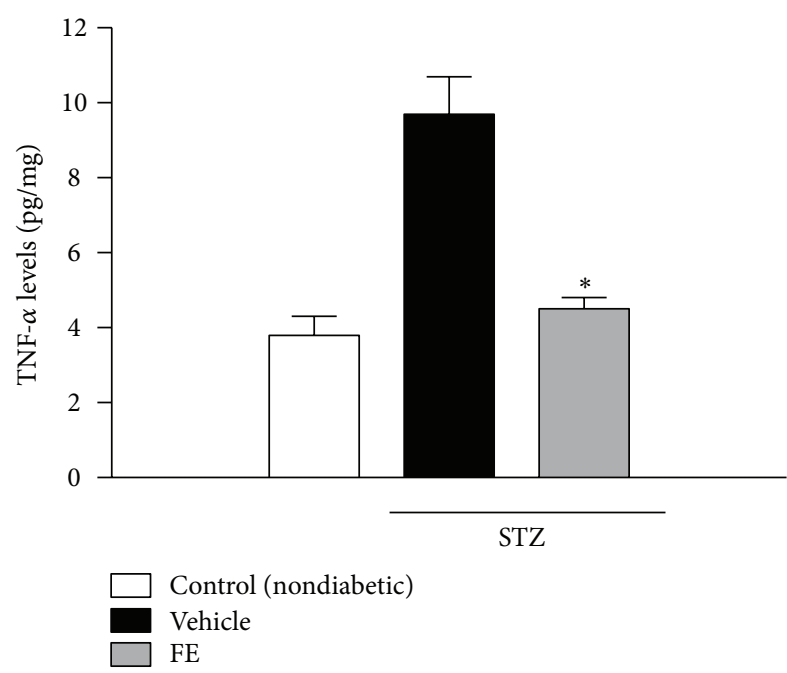

(a)

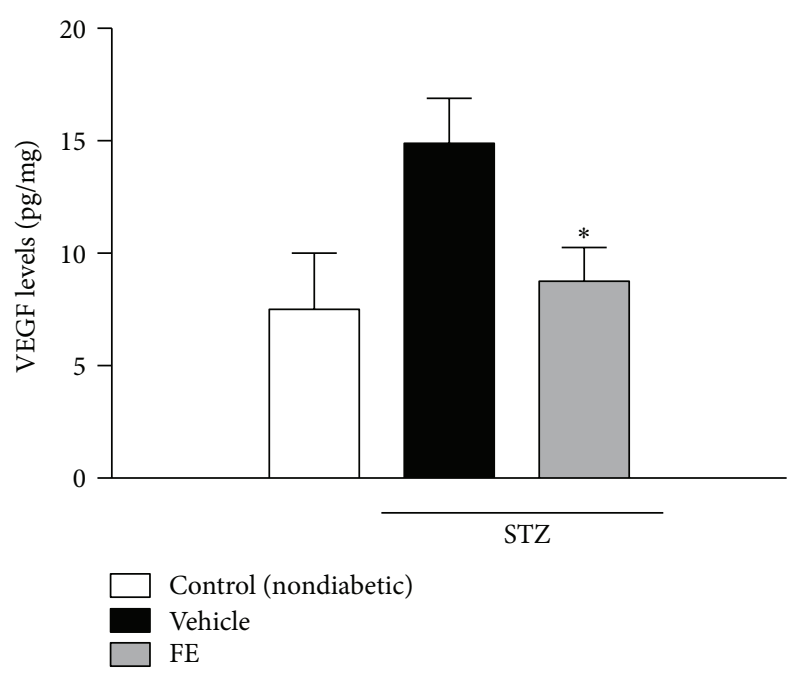

(b)

FIGURE 1: Retinal levels of TNF- $\alpha$ (a) and VEGF (b) 10 days after STZ injection with or without FE treatment. Data are expressed as the mean \pm SD. ${ }^{*} P<0.001$ versus vehicle $(n=8-10)$.

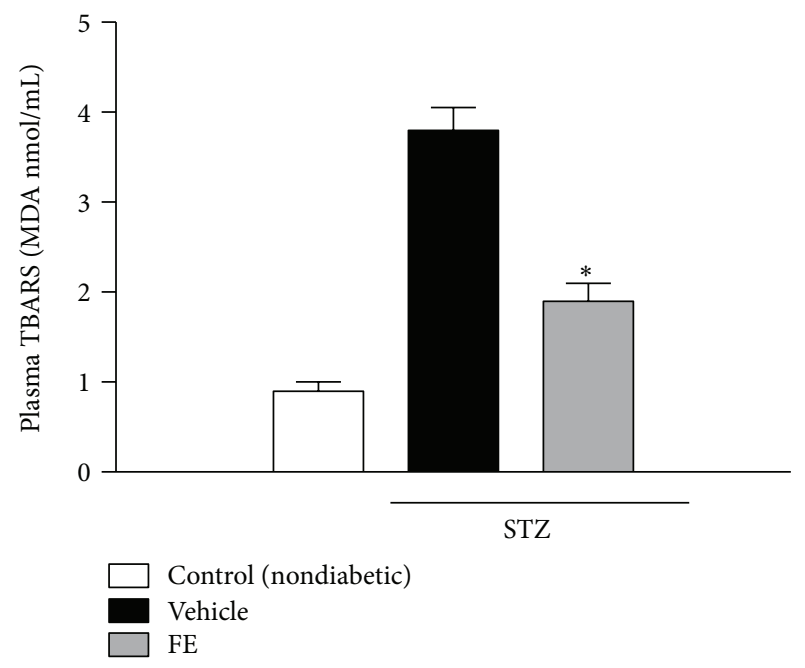

Figure 2: Lipid peroxidation. Effects of FE treatment in STZinduced diabetic rats on the formation of plasma thiobarbituric acid reactive substances (TBARS). Data are expressed as the mean \pm SD. ${ }^{*} P<0.001$ versus vehicle $(n=8-10)$.

significantly elevated in the ocular fluids such as humor vitreous and humor aqueous of patients with proliferative diabetic retinopathy [33], and many studies confirmed its primary role in the neovascularization and in the breakdown of the blood-retinal barrier $[6,34]$. We found that retinal VEGF levels increase in STZ rats and can be blunted by FE treatment (Figure 1(b)).

Oxidative stress elicited by diabetes might play a role in the development of diabetic complications [1]. Many studies have demonstrated both the crucial role of oxidative stress in the retina of diabetic animals [35] and the correlation between increased serum lipid hydroperoxides and the prevalence of retinopathy in diabetic patients [36]. Lipid peroxidation is a free radical-induced process leading to oxidative deterioration of polyunsaturated fatty acids (PUFAs). Under physiological conditions, the concentrations of plasma lipid peroxides are low. Our results show that in diabetic animals the levels of TBARS were high in the plasma and were restored to normal values after the treatment with FE.

Kowluru et al. [12] investigated the effect of long-term administration of different antioxidants on the development of retinal capillary lesions in two animal models of the early stages of diabetic retinopathy. They demonstrated in their animal model that a multiantioxidant diet that significantly inhibited (by 55-65\%) the formation of both pericyte ghosts and acellular capillaries, and significantly blunted oxidative stress. Previous studies demonstrated an increase of lipid peroxide levels in the vitreous of patients suffering from proliferative diabetic retinopathy [37]. Increased serum lipid peroxides indicate that an increased free radical activity is associated with retinopathy, with pathogenetic implications [37]. It is interesting to note that, in the present study, treatment with the complex extract mixture inhibited such oxidative stress induced by the diabetic state.

\section{Statistical Analysis}

All values are expressed as mean $\pm \mathrm{SD}$. The results were analyzed by one-way ANOVA followed by a Bonferroni post hoc test for multiple comparisons. Differences were considered statistically significant when $P$ values were less than 0.05 .

\section{Conclusions}

These data suggest that FE of red berry, GBE, and white willow bark, along with $\alpha$-lipoic acid and L-carnosine may be useful in the treatment of diabetic retinopathy and that clinical studies to evaluate this possibility are warranted. 


\section{List of Abbreviations}

VEGF: Vascular endothelial growth factor

TNF- $\alpha$ : Tumor necrosis factor-alpha

ICAM-1: Intercellular adhesion molecule-1

eNOS: Endothelial nitric oxide synthase

8-OHdG: 8-Hydroxy-2'-deoxyguanosine

NF- $\kappa$ B: Nuclear factor

kappa-light-chain-enhancer of activated

B cells

ROS: $\quad$ Reactive oxygen species

AGE: Advanced glycation end-product

RNS: $\quad$ Reactive nitrogen species

CN-1: Carnosinase

GBE: Ginkgo biloba leaf extract

TBARS: Thiobarbituric acid reactive substances

LDL: Low-density lipoprotein

STZ: $\quad$ Streptozotocin

ELISA: Enzyme-linked immunoSorbent assay

FE: $\quad$ Fortified extracts

COX-2: Cyclooxygenase enzyme-2

MDA: malonaldehyde.

\section{Conflict of Interests}

All authors have no conflict of interest or a direct financial relation with the commercial identities mentioned in the present paper.

\section{Acknowledgments}

The authors wish to thank Dr. Antony Bridgewood for his English revision of the paper. This work was supported in part by the National Grant PRIN 2009-BM7LJC and National Grant PON01-00110.

\section{References}

[1] R. A. Kowluru and P. S. Chan, "Oxidative stress and diabetic retinopathy," Experimental Diabesity Research, vol. 2007, Article ID 43603, 12 pages, 2007.

[2] L. P. Aiello, J. M. Northrup, B. A. Keyt, H. Takagi, and M. A. Iwamoto, "Hypoxic regulation of vascular endothelial growth factor in retinal cells," Archives of Ophthalmology, vol. 113, no. 12, pp. 1538-1544, 1995.

[3] G. A. Limb, L. Webster, H. Soomro, S. Janikoun, and J. Shilling, "Platelet expression of tumour necrosis factor-alpha (TNF- $\alpha$ ), TNF receptors and intercellular adhesion molecule-1 (ICAM-1) in patients with proliferative diabetic retinopathy," Clinical and Experimental Immunology, vol. 118, no. 2, pp. 213-218, 1999.

[4] J. Tang and T. S. Kern, "Inflammation in diabetic retinopathy," Progress in Retinal and Eye Research, vol. 30, no. 5, pp. 343-358, 2011.

[5] H. Wu, G. Xu, Y. Liao et al., "Supplementation with antioxidants attenuates transient worsening of retinopathy in diabetes caused by acute intensive insulin therapy," Graefe's Archive for Clinical and Experimental Ophthalmology, vol. 250, no. 10, pp. 14531458, 2012.

[6] C. Bucolo, G. M. Leggio, F. Drago, and S. Salomone, "Eriodictyol prevents early retinal and plasma abnormalities in streptozotocin-induced diabetic rats," Biochemical Pharmacology, vol. 84, no. 1, pp. 88-92, 2012.

[7] S. G. Lee, C. G. Lee, I. H. Yun, D. Y. Hur, J. W. Yang, and H. W. Kim, "Effect of lipoic acid on expression of angiogenic factors in diabetic rat retina," Clinical \& Experimental Ophthalmology, vol. 40, no. 1, pp. e47-e57, 2012.

[8] F. Pfister, E. Riedl, Q. Wang et al., "Oral carnosine supplementation prevents vascular damage in experimental diabetic retinopathy," Cellular Physiology and Biochemistry, vol. 28, no. 1, pp. 125-136, 2011.

[9] L. Packer, E. H. Witt, and H. J. Tritschler, "Alpha-lipoic acid as a biological antioxidant," Free Radical Biology and Medicine, vol. 19, no. 2, pp. 227-250, 1995.

[10] M. Kojima, L. Sun, I. Hata, Y. Sakamoto, H. Sasaki, and K. Sasaki, "Efficacy of $\alpha$-lipoic acid against diabetic cataract in rat," Japanese Journal of Ophthalmology, vol. 51, no. 1, pp. 10-13, 2007.

[11] Z. Kyselova, M. Stefek, and V. Bauer, "Pharmacological prevention of diabetic cataract," Journal of Diabetes and Its Complications, vol. 18, no. 2, pp. 129-140, 2004.

[12] R. A. Kowluru, J. Tang, and T. S. Kern, "Abnormalities of retinal metabolism in diabetes and experimental galactosemia: VII. Effect of long-term administration of antioxidants on the development of retinopathy," Diabetes, vol. 50, no. 8, pp. 1938 1942, 2001.

[13] R. A. Kowluru and S. Odenbach, "Effect of long-term administration of $\alpha$-lipoic acid on retinal capillary cell death and the development of retinopathy in diabetic rats," Diabetes, vol. 53, no. 12, pp. 3233-3238, 2004.

[14] R. A. Kowluru and M. Kanwar, "Effects of curcumin on retinal oxidative stress and inflammation in diabetes," Nutrition and Metabolism, vol. 4, article no. 8, 2007.

[15] R. A. Kowluru, M. Kanwar, and A. Kennedy, "Metabolic memory phenomenon and accumulation of peroxynitrite in retinal capillaries," Experimental Diabesity Research, vol. 2007, Article ID 21976, 7 pages, 2007.

[16] P. Pacher, I. G. Obrosova, J. G. Mabley, and C. Szabó, "Role of nitrosative stress and peroxynitrite in the pathogenesis of diabetic complications. Emerging new therapeutical strategies," Current Medicinal Chemistry, vol. 12, no. 3, pp. 267-275, 2005.

[17] S. H. Jung, K. D. Kang, D. Ji et al., "The flavonoid baicalin counteracts ischemic and oxidative insults to retinal cells and lipid peroxidation to brain membranes," Neurochemistry International, vol. 53, no. 6-8, pp. 325-337, 2008.

[18] K. Rahman, "Studies on free radicals, antioxidants, and cofactors," Clinical Interventions in Aging, vol. 2, no. 2, pp. 219-236, 2007.

[19] G. Marrazzo, P. Bosco, F. La Delia et al., "Neuroprotective effect of silibinin in diabetic mice," Neuroscience Letters, vol. 504, no. 3, pp. 252-256, 2011.

[20] C. Nizard, E. Noblesse, C. Boisdé et al., "Heat shock protein 47 expression in aged normal human fibroblasts: modulation by Salix alba extract," Annals of the New York Academy of Sciences, vol. 1019, pp. 223-227, 2004.

[21] J. J. Gagnier, M. W. van Tulder, B. Berman, and C. Bombardier, "Herbal medicine for low back pain: a Cochrane review," Spine, vol. 32, no. 1, pp. 82-92, 1976.

[22] I. Ilieva, K. Ohgami, K. Shiratori et al., "The effects of Ginkgo biloba extract on lipopolysaccharide-induced inflammation in vitro and in vivo," Experimental Eye Research, vol. 79, no. 2, pp. 181-187, 2004. 
[23] T. Yoshikawa, Y. Naito, and M. Kondo, "Ginkgo biloba leaf extract: review of biological actions and clinical applications," Antioxidants and Redox Signaling, vol. 1, no. 4, pp. 469-480, 1999.

[24] C. Bucolo, K. W. Ward, E. Mazzon, S. Cuzzocrea, and F. Drago, "Protective effects of a coumarin derivative in diabetic rats," Investigative Ophthalmology and Visual Science, vol. 50, no. 8, pp. 3846-3852, 2009.

[25] S. Faro, T. Avitabile, and G. Malaguarnera, "Effect of an antioxidant blend in diabetic macular edema," Investigative Ophthalmology \& Visual Science, vol. 54, p. 1968, 2013, EAbstract.

[26] Y. Ozawa, T. Kurihara, M. Sasaki et al., "Neural degeneration in the retina of the streptozotocin-induced type 1 diabetes model," Experimental Diabetes Research, vol. 2011, Article ID 108328, 7 pages, 2011.

[27] A. Amore and R. Coppo, "Role of apoptosis in pathogenesis and progression of renal diseases," Nephron, vol. 86, no. 2, pp. 99$104,2000$.

[28] A. B. El-Remessy, M. Al-Shabrawey, Y. Khalifa, N. T. Tsai, R. B. Caldwell, and G. I. Liou, "Neuroprotective and bloodretinal barrier-preserving effects of cannabidiol in experimental diabetes," American Journal of Pathology, vol. 168, no. 1, pp. 235$244,2006$.

[29] A. M. Joussen, V. Poulaki, N. Mitsiades et al., "Nonsteroidal anti-inflammatory drugs prevent early diabetic retinopathy via TNF-alpha suppression," The FASEB Journal, vol. 16, no. 3, pp. 438-440, 2002.

[30] S. A. Vinores, W. H. Xiao, J. Shen, and P. A. Campochiaro, "TNF- $\alpha$ is critical for ischemia-induced leukostasis, but not retinal neovascularization nor VEGF-induced leakage," Journal of Neuroimmunology, vol. 182, no. 1-2, pp. 73-79, 2007.

[31] A. M. Joussen, S. Doehmen, M. L. Le et al., "TNF- $\alpha$ mediated apoptosis plays an important role in the development of early diabetic retinopathy and long-term histopathological alterations," Molecular Vision, vol. 15, pp. 1418-1428, 2009.

[32] M. Amadio, C. Bucolo, G. M. Leggio, F. Drago, S. Govoni, and A. Pascale, "The PKC $\beta /$ HuR/VEGF pathway in diabetic retinopathy," Biochemical Pharmacology, vol. 80, no. 8, pp. 12301237, 2010.

[33] T. Awata, K. Inoue, S. Kurihara et al., "A common polymorphism in the 5/-untranslated region of the VEGF gene is associated with diabetic retinopathy in type 2 diabetes," Diabetes, vol. 51, no. 5, pp. 1635-1639, 2002.

[34] S. Rangasamy, P. G. McGuire, and A. Das, "Diabetic retinopathy and inflammation: novel therapeutic targets," Middle East African Journal of Ophthalmology, vol. 19, pp. 52-59, 2012.

[35] C. W. Karpen, K. A. Pritchard Jr., A. J. Merola, and R. V. Panganamala, "Alterations of the prostacyclin-thromboxane ratio in streptozotocin induced diabetic rats," Prostaglandins and Medicine, vol. 8, no. 2, pp. 93-103, 1982.

[36] D. Armstrong, N. Abdella, A. Salman, N. Miller, E. A. Rahman, and M. Bojancyzk, "Relationship of lipid peroxides to diabetic complications. Comparison with conventional laboratory tests," Journal of Diabetes and its Complications, vol. 6, no. 2, pp. 116122, 1992.

[37] M. E. Hartnett, R. D. Stratton, R. W. Browne, B. A. Rosner, R. J. Lanham, and D. Armstrong, "Serum markers of oxidative stress and severity of diabetic retinopathy," Diabetes Care, vol. 23, no. 2, pp. 234-240, 2000. 


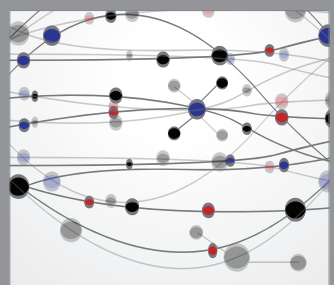

The Scientific World Journal
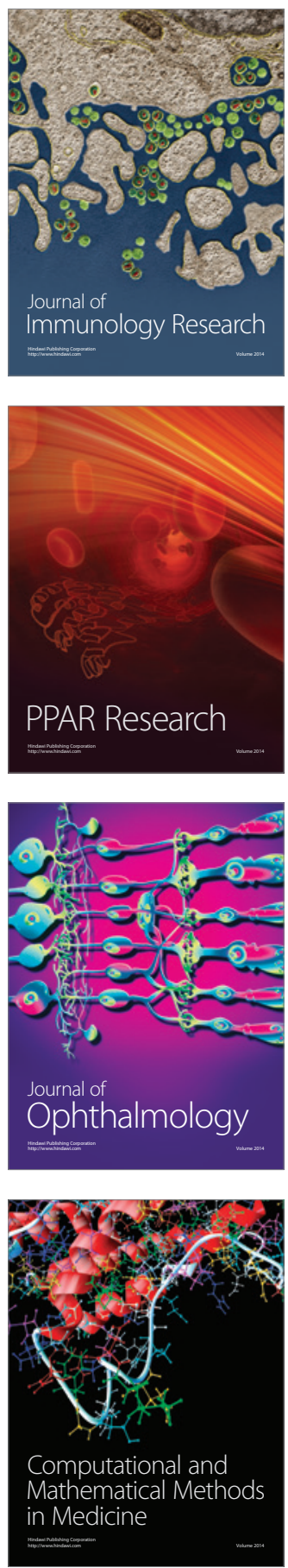

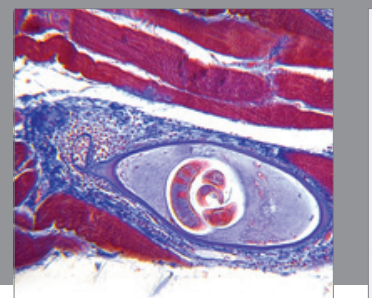

Gastroenterology

Research and Practice
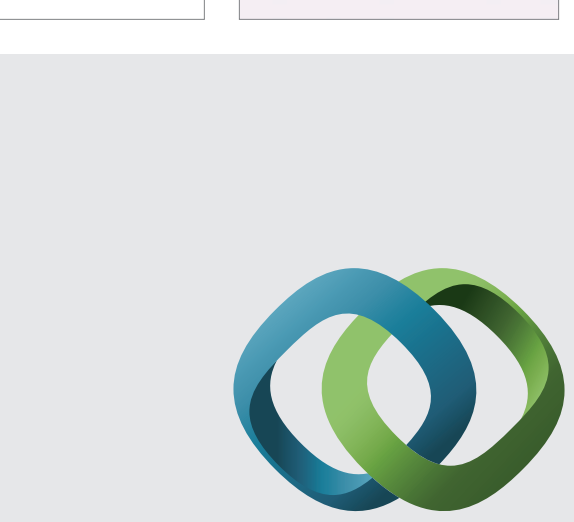

\section{Hindawi}

Submit your manuscripts at

http://www.hindawi.com
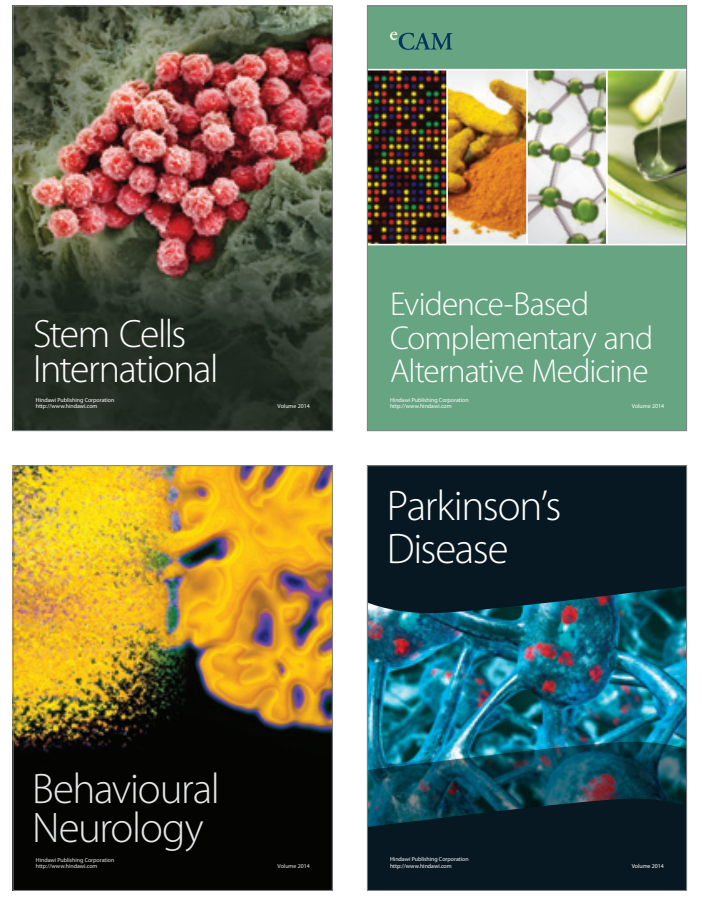
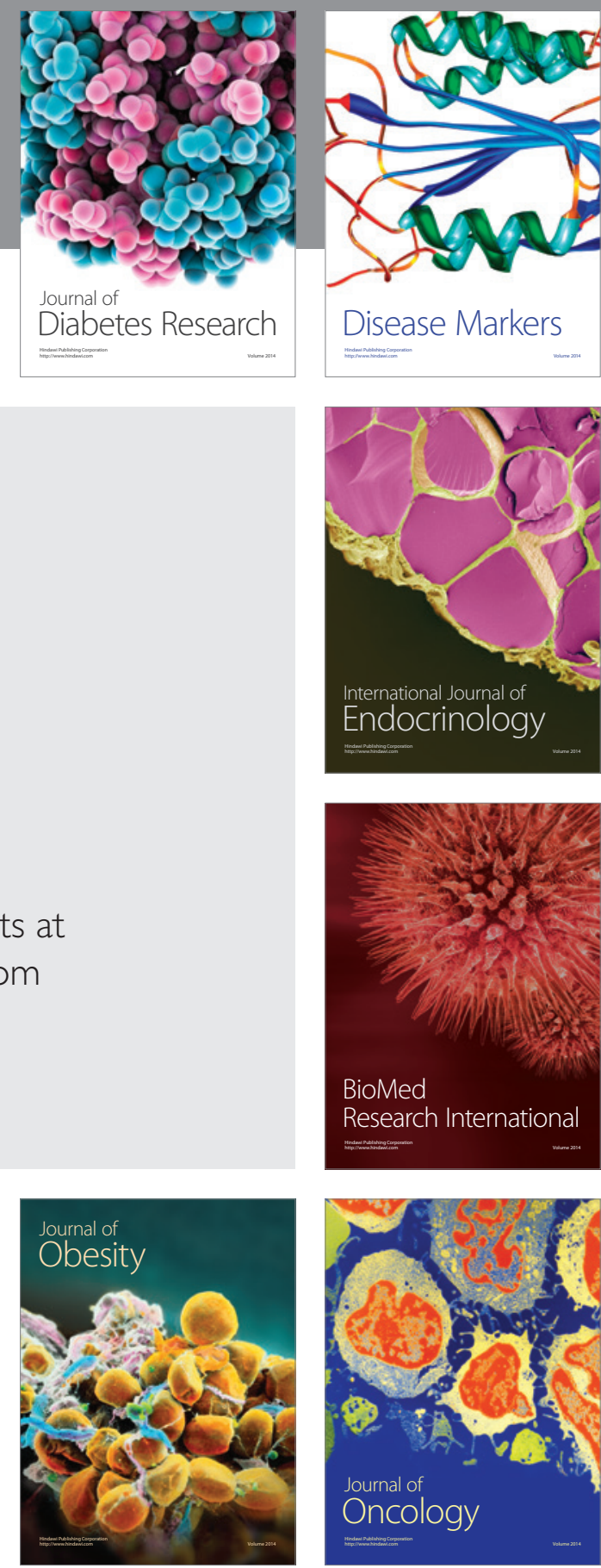

Disease Markers
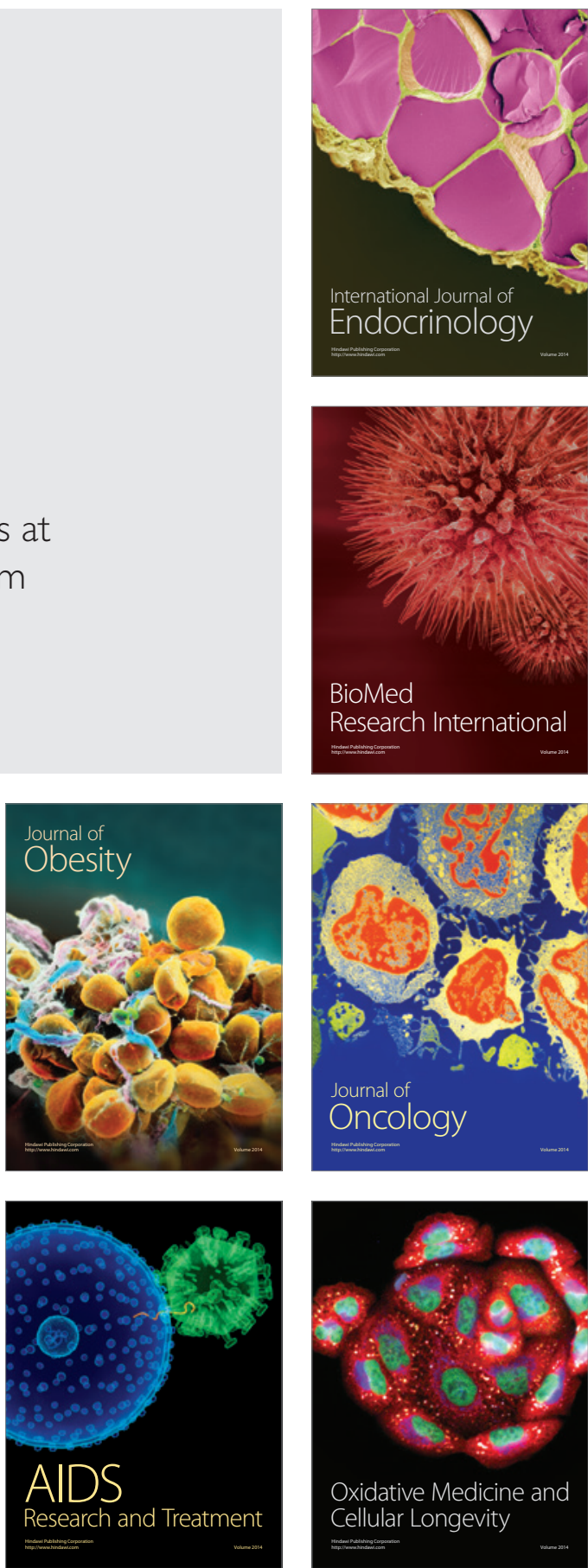